Original article

\title{
Trends, regional inequalities and determinants in the utilization of prenatal care and skilled birth attendant in Ethiopia: A multilevel analysis
}

\author{
Berhanu Teshome Woldeamanuel ", Merga Abdissa Aga \\ Salale University, Department of Statistics, Ethiopia
}

\section{A R T I C L E I N F O}

\section{Keywords:}

Prenatal care

Skilled birth attendants

Ethiopia

Maternal mortality

\begin{abstract}
A B S T R A C T
Background: Ethiopia has made a substantial reduction in maternal mortality over the last two decades. However, maternal health care utilization among 15-49 aged women remains low despite this progress. The study aimed to examine trends, regional inequalities, and potential determinants associated with prenatal care utilization and a skilled birth attendant from 2000 to 2016 in Ethiopia.

Methods: Nationally representative data extracted from the 2000 to 2016 Ethiopia Demographic and Health Surveys (EDHS), conducted every five years among 15-49 age women in Ethiopia. We used multilevel logistic analysis incorporating the demographic, socioeconomic, and health variables that are independently associated with prenatal care (PNC) and skilled birth attendant (SBA) use.

Results: The prevalence of prenatal care utilization was relatively stagnant between 2000 and 2011 but showed an overall increase nationwide from $26.7 \%$ to $62.4 \%$ between 2000 and 2016 (p-value $<0.001$ ). The prevalence of skilled birth assistance increased only by $16.5 \%$ in the past fifteen years, from $5.6 \%$ in 2000 to $22.1 \%$ in 2016 . Rural type of residence, lower maternal education, lower household wealth index, lower partner's education, an immense distance of getting medical help were steadily significantly associated with prenatal health care use and skilled birth attendants. Further, male household heads, younger age women, and no covered health insurance were associated with lower odds of skilled birth attendant use.

Conclusions: Though overall prenatal care and skilled birth attendant use prevalence have improved nationally, the regional disparity is uneven. Therefore, to sustain the progress equals more attention is needed for Afar, Somali, and Oromia regions to increase maternal health care services utilization in Ethiopia.
\end{abstract}

\section{Introduction}

Worldwide the World Health Organization (WHO) witnessed maternal mortality declined by $38 \%$ between the years 2000 and 2017 from 342 to 211 deaths per 100,000 live births due to complications linked to pregnancy and childbirth. $94 \%$ of maternal mortality occurred in low and middle-income countries. The sub-Saharan Africa region only consisted of $62 \%$ of universal mortalities, with a maternal mortality rate of 510 deaths per 100,000 live births. ${ }^{1}$ Maternal mortality has become a vital public health concern in many developing countries, particularly in sub-Saharan Africa. ${ }^{2}$ About one among 45 mothers die in low-income countries while giving birth; while in the developed regions, this figure was 1 in 5400 pregnancies. $^{3}$

In Ethiopia between 2000 and 2016, maternal mortality declined from 871 to 412 deaths per 100,000 live, still far above the global estimate. $^{4}$ In 2015 the Ethiopian government announced and implemented Health Sector Transformation Plan (HSTP), ${ }^{5}$ aimed to reduce the maternal mortality ratio to 199 deaths per 100,000 live births and the neonatal mortality rate to 10 per 1000 live births by 2020 . It can achieve through improving delivery at a health facility, with skilled medical attention and hygienic conditions; reduction in complications and infections during labor and delivery; on-time postnatal care that luxuries difficulties from delivery; and education of the mother on care for herself and her newborn. Despite these policies and intervention efforts to achieve the millennium development goals, maternal mortality is still very high in Ethiopia.

In Ethiopia, maternal mortality is one of the leading required health and welfare issues among women aged 15-49 years old. ${ }^{6}$ Whatsoever the economic development and the extensive reduction of impoverishment within the past two decades within the country, maternal mortality remains at a more significant level and stays a severe public health issue. The maternal mortality rate of $58,51,44$, and $38.4 \%$ reported in 2000 ,

\footnotetext{
* Corresponding author.

E-mail addresses: berteshome@gmail.com (B.T. Woldeamanuel), mergabdisa3@gmail.com (M.A. Aga).
} 
2005, 2011, and 2016 in Ethiopia, respectively. ${ }^{3,7-9}$ Complications of pregnancy are the leading causes of death among mothers aged 15-49 years old in Africa and other developing countries. Hence, better quality prenatal care and a skilled attendant are vital to managing complications during delivery. ${ }^{10}$

Corresponding to other sub-Saharan countries, prenatal and skilled birth attendant care utilization regional disparity is very high in Ethiopia. However, regions of the same economic status have various health care distribution. The trouble of unfair distribution of health care services needs particular attention, with evidence of the country's emerging regions having the worst health care uses. ${ }^{4}$

In Ethiopia, the percentage of deliveries assisted by a skilled birth attendant (SBA) increased from 5.6 to $27.7 \%$ from $2000-2016 .{ }^{4,7-9}$ It is comparatively shy relative to the rise in the rate of pregnant women receiving prenatal visits, which have increased from 26.7 to $62.4 \%$ for the duration of the same period. Antenatal visits also offer a window of chance to probe and perhaps avoid adverse birth events. Evidence advocates that there is significant inequality in the admittance to vital health services, counting services for pregnancy and childbirth. ${ }^{11}$

We examined secondary data from the four National Demographic and Health Survey (DHS) data 2000-2016 to investigate the regional inequality in prenatal care and skilled birth attendant utilization at a national and regional level in Ethiopia. Besides examining the predictors of prenatal care and skilled birth attendant utilization, we need to identify the variables that are consistently significant during the study periods. Therefore, this study aimed to determine the critical determinants of prenatal care utilization and skilled birth attendants using 2000-2016 DHS data in Ethiopia. It may help evaluate the Sustainable Development Goals (SDGs) target of reducing maternal mortality to less than 70 deaths per 100,000 live births and neonatal mortality to less than 12 deaths per 1000 live births by 2030 and taking the specific interventions. $^{12}$

\section{Methods}

\subsection{Study setting and population}

The data for the study driven from Ethiopian four DHS from 20002016. ${ }^{4,7-9}$ The EDHS was a nationwide cross-sectional analysis conducted by the Central Statistical Agency (CSA), the Ministry of Health $(\mathrm{MoH})$, and the Ethiopian Public Health Institute every five years, where the United States Agency for International Development (USAID) provided financial and technical support.

\subsection{Sampling}

Ethiopian 2000, 2005, 2011, and 2016 DHS included 540 enumeration areas (EAs) (139 in the urban areas and 401 in the rural), 540 EAs (145 in the urban and 395 in the rural), 624 EAs, (187 in the urban and 437 in the rural), respectively. The respondents were selected using a stratified two-stage cluster design, each region stratified into urban and rural areas. First was selecting clusters/EAs with probability proportional to enumeration area size and independent selection within each stratum. In all the selected EAs, the household listing was done. At the second stage, fixed numbers of households were selected per cluster with an equal probability systematic selection involving eligible women aged 15-49. A representative sample of 15,367, 14,070, 16,515, and 15,683 illegible women aged 15-49 with a response rate of 97.8, 96, 95, and $94.6 \%$ were interviewed from 2000, 2005, 2010, and 2016 DHS, respectively. Complete information was not available for all women in each DHS, and PNC and SBA information was missing. The final sample included 28,695 women aged 15-49 years after excluding the missing observations.

\subsubsection{Data collection}

The EDHS used three questionnaires: the Household Questionnaire, the Woman's Questionnaire, and Man's Questionnaire. These questionnaires were adapted from model survey instruments developed for the MEASURE DHS project to reflect the population and health issues relevant to Ethiopia. The national survey has information on a range of socioeconomic and demographic factors of the population. The questionnaire contains all information about the individual woman on prenatal care, delivery, an assistant during delivery, and other characteristics mother and the household. All women of 15-49 years old, who were permanent inhabitants or visitors who lived at least one night in the household before the survey, were eligible for the interview. Data were collected by conducting face-to-face interviews with women that met the eligibility criteria.

\subsection{Variables}

The Ethiopian DHS collects data on different demographic, socioeconomic, and health-related characteristics of mothers and households. In the current study, we used two outcome variables; prenatal care (PNC) service during pregnancy and professional skilled birth attendant (SBA) service utilization at birth extracted from the Ethiopian DHS, 2000, 2005, 2011, and 2016. ${ }^{4,7-9}$ PNC is a measure of skilled pregnancy care received by women during the most recent pregnancy, defined as one if a woman received the WHO recommended at least four visits and 0 elsewhere. SBA is measured as professional skilled birth assistance defined as a binary outcome for 1 , if a woman received skilled birth attendant and 0 , if otherwise.

The main predictors were woman's education, partner's education, woman age, wealth index, household head sex, residence, the region, media, employment status, the problem of getting medical help for self, covered health insurance, and parity captured from the literature on the subject. $^{13-22}$

\subsection{Ethical considerations}

The DHS data were available from MEASURE DHS for free. All EDHS surveys are approved by the Inner City Fund (ICF) International the USA and Ethiopian $\mathrm{MOH}$, and Ethiopian public health institute (EPHI) to ensure that the protocols comply with the US Division of Health and Human Services regulations for the fortification of human issues.

\subsection{Statistical analysis}

STATA (version 15.0) statistical software package used to conduct data analysis, and results were considered statistically significant if $p<$ 0.05 . Sample weights were used to account for the impact of the underlying complex sampling design on logistic regression parameters for unequal probability sampling and non-response using the svyset STATA survey commands. A post hoc power analysis was made using a 0.05 level of significance and $80 \%$ power, with various intraclass correlation coefficients (ICC). The results showed that the power was at least $85 \%$ for almost all the key variables (see Table S1, Additional file 1). We used descriptive statistics to describe study subjects' characteristics.

The two-level structure multilevel logistic regression with women at first level units and regions as second-level units have been used. Women selected randomly from the same region are assumed to share more similar characters than women selected randomly from different regions. Three multilevel logistic regression models: an empty model with random intercept, random intercept with the fixed effects model, and random coefficient with a random intercept model has been fitted. In the first step, the heterogeneity of proportions between regions in a multilevel analysis was tested using the likelihood ratio test (LRT) and Deviance test. Finally, the odds ratio (OR) with a 95\% confidence interval (CI) was reported using STATA statistical package version 15 , and p-values less than 0.05 were considered to be statistically significant. All significant variables in the unadjusted analysis (p-value $<0.05$ ) were included in the multivariable multilevel logistic model using the 
forwards fitting approach.

Let $\mathrm{p}_{\mathrm{ij}}$ denote the probability that the response variables equal 1 , $\mathrm{P}_{\mathrm{ij}}=P\left(y_{\mathrm{ij}}=1 / x_{i j}\right)$ the probability of ith woman received prenatal care or skilled birth assistance during delivery in the jth region. The two-level logistic regression model can be given as:

$\operatorname{logit}\left(P_{i j}\right)=\log \left[\frac{P_{i j}}{1-P_{i j}}\right]=\beta_{0}+\beta_{1} X_{\mathrm{ij}}+U_{o j}$

where $U_{o j}$ a random quantity and follows a normal distribution with mean zero and variance $\sigma_{u}^{2}$. This model can be split into two models; one for level 1 and the other for level2

$\operatorname{logit}\left(\mathrm{P}_{\mathrm{ij}}\right)=\log \left[\frac{P_{i j}}{1-P_{i j}}\right]=\beta_{\mathrm{oj}}+\beta_{1} \mathrm{X}_{\mathrm{ij}}[$

Model: level 1, random intercept model]

$$
\beta_{\mathrm{oj}}=\beta_{\mathrm{o}}+\mathrm{U}_{\mathrm{oj}}[
$$

Model: level 2, empty model]

where. $\mathrm{P}_{\mathrm{ij}}=\frac{e^{\beta_{O}+\sum_{h=1}^{\beta_{h} \mathrm{X}_{h i j}+\mathrm{U}_{o j}}}}{1+e^{\beta_{O}+\sum_{h=1}^{k} \beta_{h} \mathrm{X}_{h i j}+\mathrm{U}_{o j}}}$
Thus, $\quad \operatorname{logit}\left(\mathrm{P}_{\mathrm{ij}}\right)=\log \left[\frac{P_{i j}}{1-P_{i j}}\right]=\beta_{o}+\sum_{h=1}^{k} \beta_{h} x_{i j}+U_{o j}+$ $\sum_{h=1}^{k} U_{h j} x_{i j}$ the random intercept multilevel logistic regression model, the first part $\beta_{\mathrm{o}}+\sum_{h=1}^{k} \beta_{\mathrm{h}} \mathrm{X}_{\mathrm{ij}}$ is called the fixed part of the model, and the second part, $\mathrm{U}_{\mathrm{oj}}+\sum_{\mathrm{h}=1}^{\mathrm{k}} \mathrm{U}_{\mathrm{hj}} \mathrm{X}_{\mathrm{ij}}$ is called the random part of the model. The random variables or effects, $U_{0 j}, U_{1 j}, \ldots, U_{k j}$ a re assumed to be independent between groups but may be correlated within groups. So the components of the vector $\mathrm{U}_{0 \mathrm{j}}, \mathrm{U}_{1 \mathrm{j}}, \ldots, \mathrm{U}_{\mathrm{kj}}$ are independently distributed as a multivariate normal distribution with zero mean vector and variances and co-variances matrix $\Omega$.

Then the intraclass correlation (ICC) at the regions level is given by $I C C=\frac{\sigma_{\mu}^{2}}{\sigma_{\mu}^{2}+\sigma_{e}^{2}}$

Where $\sigma_{\mu}^{2} \mathrm{~s}$ the between group variance and $\sigma_{e}^{2}$ is the within group variance.

Table 1

Demographic and socioeconomic characteristics of study populations, Ethiopian DHS 2000-2016.

\begin{tabular}{|c|c|c|c|c|c|c|c|c|c|}
\hline \multirow[t]{2}{*}{ Background characteristics } & \multirow[t]{2}{*}{ Categories } & \multicolumn{2}{|c|}{$2000(\mathrm{n}=7178)$} & \multicolumn{2}{|c|}{$2005(n=6581)$} & \multicolumn{2}{|c|}{$2011(\mathrm{n}=7743)$} & \multicolumn{2}{|c|}{$2016(n=7193)$} \\
\hline & & $\mathrm{n}$ & $\%$ & $\mathrm{n}$ & $\%$ & $\mathrm{n}$ & $\%$ & $\mathrm{n}$ & $\%$ \\
\hline \multirow[t]{11}{*}{ Region } & Tigray & 1306 & 8.5 & 1257 & 8.9 & 1728 & 10.5 & 1682 & 10.7 \\
\hline & Affar & 858 & 5.6 & 789 & 5.6 & 1291 & 7.8 & 1128 & 7.2 \\
\hline & Amhara & 1909 & 12.4 & 1943 & 13.8 & 2087 & 12.6 & 1719 & 11.0 \\
\hline & Oromia & 2578 & 16.8 & 2230 & 15.8 & 2135 & 12.9 & 1892 & 12.1 \\
\hline & Somali & 844 & 5.5 & 669 & 4.8 & 914 & 5.5 & 1391 & 8.9 \\
\hline & Benishangul-gumuz & 992 & 6.5 & 846 & 6.0 & 1259 & 7.6 & 1126 & 7.2 \\
\hline & SNNP & 2028 & 13.2 & 2087 & 14.8 & 2034 & 12.3 & 1849 & 11.8 \\
\hline & Gambela & 876 & 5.7 & 729 & 5.2 & 1130 & 6.8 & 1035 & 6.6 \\
\hline & Harari & 908 & 5.9 & 844 & 6.0 & 1101 & 6.7 & 906 & 5.8 \\
\hline & Addis Ababa & 2015 & 13.1 & 1869 & 13.3 & 1741 & 10.5 & 1824 & 11.6 \\
\hline & Dire Dawa & 1053 & 6.9 & 807 & 5.7 & 1095 & 6.6 & 1131 & 7.2 \\
\hline \multirow[t]{2}{*}{ Place of residence } & Urban & 4543 & 29.6 & 4423 & 31.4 & 5329 & 32.3 & 5348 & 34.1 \\
\hline & Rural & 10,824 & 70.4 & 9647 & 68.6 & 11,186 & 67.7 & 10,335 & 65.9 \\
\hline \multirow[t]{4}{*}{ Mother education } & No education & 10,586 & 68.9 & 8454 & 60.1 & 8278 & 50.1 & 7033 & 44.8 \\
\hline & Primary & 2530 & 16.5 & 2966 & 21.1 & 5858 & 35.5 & 5213 & 33.2 \\
\hline & Secondary & 2092 & 13.6 & 2292 & 16.3 & 1395 & 8.4 & 2238 & 14.3 \\
\hline & Higher & 159 & 1.0 & 358 & 2.5 & 984 & 6.0 & 1199 & 7.6 \\
\hline \multirow[t]{5}{*}{ Father education } & No education & 7157 & 63.0 & 5946 & 58.3 & 5905 & 48.9 & 4431 & 45.1 \\
\hline & Primary & 2200 & 19.4 & 2287 & 22.4 & 4088 & 33.8 & 3054 & 31.1 \\
\hline & Secondary & 1577 & 13.9 & 1559 & 15.3 & 1160 & 9.6 & 1226 & 12.5 \\
\hline & Higher & 331 & 2.9 & 367 & 3.6 & 767 & 6.3 & 1020 & 10.4 \\
\hline & Don't know & 102 & 0.9 & 46 & 0.5 & 159 & 1.3 & 93 & $0.9 \%$ \\
\hline \multirow[t]{5}{*}{ Wealth index } & Poorest & - & - & 2738 & 19.5 & 3711 & 22.5 & 3894 & 24.8 \\
\hline & Poorer & - & - & 2080 & 14.8 & 2402 & 14.5 & 2046 & 13.0 \\
\hline & Middle & - & - & 2051 & 14.6 & 2268 & 13.7 & 2002 & 12.8 \\
\hline & Richer & - & - & 1958 & 13.9 & 2505 & 15.2 & 2042 & 13.0 \\
\hline & Richest & - & - & 5243 & 37.3 & 5629 & 34.1 & 5699 & 36.0 \\
\hline \multirow[t]{2}{*}{ Mother work status } & No working & 6830 & 44.5 & 9919 & 70.5 & 10,557 & 64.0 & 10,011 & 63.8 \\
\hline & Working & 8528 & 55.5 & 4143 & 29.5 & 5938 & 36.0 & 5672 & 36.2 \\
\hline \multirow[t]{2}{*}{ Sex of household head } & Male & 11,585 & 75.4 & 10,512 & 74.7 & 11,889 & 72.0 & 10,853 & 69.2 \\
\hline & Female & 3782 & 24.6 & 3558 & 25.3 & 4626 & 28.0 & 4830 & 30.8 \\
\hline \multirow[t]{3}{*}{ Mother age } & $15-19$ & 3584 & 23.3 & 3252 & 23.1 & 3835 & 23.2 & 3498 & 22.3 \\
\hline & $20-34$ & 7462 & 48.6 & 6928 & 49.2 & 8307 & 50.3 & 7989 & 50.9 \\
\hline & $35-49$ & 4321 & 28.1 & 3890 & 27.6 & 4373 & 26.5 & 4196 & 26.8 \\
\hline \multirow[t]{2}{*}{ Marital status } & Never married/divorced/widowed & 5987 & 39.0 & 5426 & 38.6 & 6311 & 38.2 & 5859 & 37.4 \\
\hline & Married/living together & 9380 & 61.0 & 8644 & 61.4 & 10,204 & 61.8 & 9824 & 62.6 \\
\hline \multirow[t]{4}{*}{ Birth in the last of five years } & 1 & 4074 & 26.5 & 3731 & 26.5 & 4482 & 27.1 & 4278 & 27.3 \\
\hline & 2 & 2752 & 17.9 & 2481 & 17.6 & 2722 & 16.5 & 2426 & 15.5 \\
\hline & 3 & 385 & 2.5 & 344 & 2.4 & 513 & 3.1 & 447 & 2.9 \\
\hline & 4 & 30 & 0.2 & 30 & 0.2 & 46 & 0.3 & 40 & 0.3 \\
\hline \multirow[t]{3}{*}{ Parity } & 1 & 1765 & 17.4 & 1620 & 17.3 & 2015 & 18.5 & 1974 & 19.2 \\
\hline & $2-4$ & 4015 & 39.6 & 3862 & 41.4 & 4552 & 41.8 & 4387 & 42.7 \\
\hline & $5+$ & 4363 & 43.0 & 3857 & 41.3 & 4329 & 39.7 & 3913 & 38.1 \\
\hline \multirow{2}{*}{ Distance } & Big problem & - & - & 8456 & 60.1 & 10,412 & 63.1 & 4341 & 27.7 \\
\hline & Not a big problem & - & - & 5609 & 39.9 & 6090 & 36.9 & 11,342 & 72.3 \\
\hline
\end{tabular}




\section{Results}

\subsection{Study population}

Table 1 summarizes the characteristics of the study population 2000-2016 Ethiopian DHS. The proportion of study participants vary across regions, Oromia, South Nations Nationalities and People, Addis Ababa and Amhara regions with the highest proportion of study participants in all survey. Also, a study participant is predominantly rural across the surveys. The percentage of women and partners with no education declines from $69 \%$ to $44.8 \%$ and from $63 \%$ to $45.1 \%$, respectively. However, that of women with secondary and higher education has not significantly increased. About one-fourth of the respondents gave only one birth in the past five years, across all surveys $(26.5 \%-$ $27.3 \%$ ). Also, most women have at least 5 five children in 2000 and 2-4 children in the recent three surveys (2005-2016).

The general pattern of age showed most women were between 20 and 34 years old across all surveys. In 2005 and 2011, most women reported a big problem getting medical help for themselves (Table 1).

\subsection{Trend in the prevalence of prenatal care utilization and skilled birth assistant in Ethiopia from 2000 to 2016}

The total number of women included in each survey 2000, 2005, 2011, and 2016 were $7178,6581,7743$, and 7193, respectively. The prevalence of prenatal care service utilization in the four survey years was $26.7 \%$, in $2000,27.69 \%$ in $2005,33.9 \%$ in 2011 , and $62.4 \%$, whereas $4871(31.7 \%), 4481(68.1 \%), 4828(62.4 \%)$ and $3140(43.7 \%)$ of mothers who gave birth in each survey year received skilled birth attendant during delivery. The prevalence of prenatal care utilization among pregnant mothers in Ethiopia was relatively stagnant between 2000 and 2011 but showed an overall increase nationwide from $26.7 \%$ in 2000 to $62.4 \%$ in 2016 (p-value < 0.001 ) (Table 2). The prevalence of skilled birth assistance increased only by $16.5 \%$ in the past fifteen years, from $5.6 \%$ in 2000 to $22.1 \%$ in 2016 .

\subsection{Bivariate analysis: prevalence of prenatal care utilization by household and maternal characteristics}

The prevalence of prenatal care utilization persistently advanced among women from the Harari, Dire Dawa, and Addis Ababa cities are remaining relatively higher between 2000 and 2016 (50.2\% vs. 75.9\%, $57.6 \%$ vs. $87.4 \%$, and $83.1 \%$ vs. $96.8 \%$, respectively). On the other hand, prenatal care utilization trends among women from the Tigray region showed significant radical increases between 2000 and 2016 (36.4\% vs. 90\%). Women in the Somali region revealed overall persistent lower prevalence prenatal care utilization all through that same period (14.6\% vs. $43.6 \%$ ). Further, women from Afar and Oromia reported the lowest percentage prenatal care utilization $(51.3 \%$ and $50.7 \%$ ), which relatively lower increases between 2000 and 2016 ( $26.1 \%$ vs. $51.3 \%$ and $27 \%$ vs. $50.7 \%$, respectively). The economic differential prenatal care utilization among pregnant women in the poorest wealth index families increased from $12.7 \%$ in 2005 to $47.5 \%$ in 2016, while it was increased from $58 \%$ in 2005 to $84.8 \%$ in 2016 . It means that the prevalence of prenatal care utilization among women from the poorest household was still less than that richest household utilization in 2005 (47.5\% vs. 58\%).

Likewise, prenatal care utilization was more customary among those women living in urban areas relative to rural residents between 2000 and 2016 (66.6\% vs. $90.1 \%$ and $21.6 \%$ vs. $51.3 \%$, respectively). As maternal characteristics were concerned, the prevalence of prenatal care utilization was significantly higher among women with at least secondary educated throughout all year relative to women who had no education or primary education levels (Table 2). Prenatal care utilization was also significantly higher among women whose partner had attained higher education relative to those whose partner had no education changes from $77.4 \%$ in 2000 to $82.3 \%$ in 2016 for higher education level partner to $21.1 \%$ in 2000 to $43.0 \%$ in 2016 in a noneducated partner.

Concerning distances (the difficulties of getting medical help for self), prenatal care utilization was higher among women with not a big problem of getting medical help (ranging from 50.4\% in 2000 to $61.3 \%$ in 2016) relative to those with a big issue of getting medical help for self. The prevalence of prenatal care utilization was highest among women who had only one child ever born or only child born in the past five years before each survey (31.5\% vs. $61.2 \%$ between 2000 and 2016 and $42.1 \%$ vs. $70.9 \%$ between 20002016 , respectively) relative to those mothers ever born at least five children. Further, working women and women heads of households had a greater prevalence of prenatal care utilization relative to not working and whose household head was male across all survey years (Table 2).

\subsection{Bivariate analysis: prevalence of skilled birth attendant utilization by household and maternal characteristics}

Table 2 also indicates that the prevalence of skilled birth assistance has been steadily increasing over the last four surveys. The prevalence of skilled birth assistants increased from 5.6\% in 2000 to $10 \%$ in 2011 and $27.7 \%$ in 2016, 22.1\% between 2000 and 2016. The magnitude of increase in the prevalence of skilled birth assistance varies by region, resident, and maternal characteristics between 2000 and 2016. The highest prevalence increase was observed in Tigray ( $4.8 \%$ vs. $59.5 \%$ ), Gambella (23.8\% vs. $46.9 \%$ ), Harari (26\% vs. $51.2 \%$ ) regions and Dire Dawa (33.5\% vs. 56.7\%) and Addis Ababa (69.1\% vs. 96.8\%) cities between 2000 and 2016. However, the regions Afar (5.5\% vs. 16.4\%), Oromia (4.9\% vs. $19.7 \%)$, and Somali $(7.2 \%$ vs. $20 \%)$ indicated an overall persistent lower prevalence of skilled birth assistants during that same period.

Mother's educational status has a significant impact on increasing utilization of skilled birth assistance during delivery. Higher education is associated with a higher prevalence of skilled birth assistance during delivery all over the four surveys relative to no education. The current study indicated that women with higher education or secondary have a higher prevalence of skilled birth assistance during birth. Concerning the partner's education status, the analysis shows that women whose partner is educated have an increased prevalence of a skilled birth assistant than that of whose partner is uneducated from 2000 to 2016 . The household wealth index was another variable that shows a variation in skilled birth assistants in the country. The investigation revealed a higher prevalence of skilled birth assistants among women from the richest wealth index ( $26.6 \%$ vs. $70.3 \%$ between 2005 and) than women belongs the poorest household ( $0.7 \%$ vs. $11 \%$ between 2005 and 2016 ).

The type of residence was another household characteristic associated with the trend of skilled birth assistants at birth. The study showed a higher persistent increase in skilled birth assistants among women from urban (34\% versus $80.1 \%$ ) than for rural (2.3\% versus $21.2 \%$ ) women between 2000 and 2016. The distance was another vital predictor variable found significantly associated with a skilled birth assistant during delivery in this study. The highest prevalence of skilled birth assistant observed among women who had a big problem getting medical help for self.

In comparison, the increase in the prevalence of skilled birth assistants during delivery was consistent among mothers of different age groups. The sex of the household head is essential to determine the mother's decision-making autonomy of the mother's utilization of skilled birth assistants. This study revealed an increased prevalence of skilled birth assistants in women who are heads of a household.

\subsection{Multivariable multilevel analysis of factors associated with prenatal care utilization}

Table 3 presents the multivariable multilevel logistic regression 
Table 2

Prevalence of prenatal care and skilled birth attendant services by selected variables, Ethiopia 2000-2016.

\begin{tabular}{|c|c|c|c|c|c|c|c|c|c|c|c|}
\hline \multirow[t]{2}{*}{$\begin{array}{l}\text { Background } \\
\text { characteristics }\end{array}$} & \multirow[t]{2}{*}{ Categories } & \multicolumn{4}{|c|}{$\begin{array}{l}\text { Number (percent) of women received } \\
\text { prenatal care }\end{array}$} & \multirow[t]{2}{*}{$\begin{array}{l}\text { \% Change during } \\
2000-2016\end{array}$} & \multicolumn{4}{|c|}{$\begin{array}{l}\text { Number (percent) of women received with } \\
\text { skilled birth attendant }\end{array}$} & \multirow[t]{2}{*}{$\begin{array}{l}\text { \% Change during } \\
2000-2016\end{array}$} \\
\hline & & 2000 & 2005 & 2011 & 2016 & & 2000 & 2005 & 2011 & 2016 & \\
\hline & Overall & 26.7 & 27.6 & 33.9 & 62.4 & 35.7 & 5.6 & 5.7 & 10 & 27.7 & 22.1 \\
\hline \multirow[t]{12}{*}{ Region } & Tigray & 252 & 240 & 415 & 634 & 53.6 & 25 & $41(6)$ & 103 & 483 & 54.5 \\
\hline & Acf & $(36.4)$ & (35.3) & $(50.1)$ & (90) & & $(4.8)$ & & (11.6) & (59.3) & \\
\hline & Affar & $\begin{array}{l}100 \\
(26.1)\end{array}$ & $46(15)$ & $\begin{array}{l}188 \\
(32.3)\end{array}$ & $\begin{array}{l}283 \\
(51.3)\end{array}$ & 25.2 & $\begin{array}{l}18 \\
(5.5)\end{array}$ & $\begin{array}{l}14 \\
(4.5)\end{array}$ & $41(7.2)$ & $\begin{array}{l}89 \\
(16.4)\end{array}$ & 10.9 \\
\hline & Amhara & $\begin{array}{l}209 \\
(18.9)\end{array}$ & $\begin{array}{l}276 \\
(26.5)\end{array}$ & $\begin{array}{l}297 \\
(33.6)\end{array}$ & $\begin{array}{l}415 \\
(67.1)\end{array}$ & 48.2 & $\begin{array}{l}38 \\
(3.1)\end{array}$ & $\begin{array}{l}47 \\
(3.7)\end{array}$ & $\begin{array}{l}85 \\
(10.1)\end{array}$ & $\begin{array}{l}209 \\
(27.7)\end{array}$ & 24.6 \\
\hline & Oromia & $\begin{array}{l}361 \\
(27)\end{array}$ & $\begin{array}{l}307 \\
(24.8)\end{array}$ & $\begin{array}{l}342 \\
(31.3)\end{array}$ & $\begin{array}{l}384 \\
(50.7)\end{array}$ & 23.7 & $\begin{array}{l}45 \\
(4.9)\end{array}$ & $\begin{array}{l}62 \\
(4.8)\end{array}$ & $\begin{array}{l}104 \\
(8.1)\end{array}$ & $\begin{array}{l}250 \\
(19.7)\end{array}$ & 14.8 \\
\hline & Somali & $\begin{array}{l}53 \\
(14.6)\end{array}$ & $30(7.4)$ & $\begin{array}{l}121 \\
(21.5)\end{array}$ & $\begin{array}{l}333 \\
(43.6\end{array}$ & 29 & $\begin{array}{l}23 \\
(7.2)\end{array}$ & $\begin{array}{l}18 \\
(5.2)\end{array}$ & $50(8.4)$ & $\begin{array}{l}160 \\
(20)\end{array}$ & 12.8 \\
\hline & Benishangul & $\begin{array}{l}126 \\
(25.7)\end{array}$ & $\begin{array}{l}128 \\
(24.5)\end{array}$ & $\begin{array}{l}215 \\
(35.1)\end{array}$ & $\begin{array}{l}287 \\
(68.7)\end{array}$ & 43 & $\begin{array}{l}33 \\
(9.1)\end{array}$ & $\begin{array}{l}34 \\
(5.1)\end{array}$ & $54(8.9)$ & $\begin{array}{l}171 \\
(28.6)\end{array}$ & 19.5 \\
\hline & SNNP & $\begin{array}{l}296 \\
(28.7)\end{array}$ & $\begin{array}{l}367 \\
(30.3)\end{array}$ & $\begin{array}{l}275 \\
(27.3)\end{array}$ & $\begin{array}{l}437 \\
(69.3)\end{array}$ & 40.6 & $\begin{array}{l}39 \\
(4.9)\end{array}$ & $\begin{array}{l}56 \\
(4.2)\end{array}$ & $69(6.1)$ & $\begin{array}{l}288 \\
(28.6)\end{array}$ & 23.7 \\
\hline & Gambela & $\begin{array}{l}202 \\
(29.8)\end{array}$ & $\begin{array}{l}147 \\
(36.6)\end{array}$ & $\begin{array}{l}254 \\
(54.5)\end{array}$ & $\begin{array}{l}302 \\
(72.3)\end{array}$ & 42.5 & $\begin{array}{l}70 \\
(23.8)\end{array}$ & $\begin{array}{l}76 \\
(15.3)\end{array}$ & $\begin{array}{l}113 \\
(27.4)\end{array}$ & $\begin{array}{l}200 \\
(46.9)\end{array}$ & 23.1 \\
\hline & Harari & $\begin{array}{l}174 \\
(50.2)\end{array}$ & $\begin{array}{l}136 \\
(40.7)\end{array}$ & $\begin{array}{l}242 \\
(55.9)\end{array}$ & $\begin{array}{l}299 \\
(75.9)\end{array}$ & 25.7 & $\begin{array}{l}110 \\
(26)\end{array}$ & $\begin{array}{l}115 \\
(31.4)\end{array}$ & $\begin{array}{l}165 \\
(32.5)\end{array}$ & $\begin{array}{l}245 \\
(51.2)\end{array}$ & 25.2 \\
\hline & Addis Ababa & $\begin{array}{l}336 \\
(83.1)\end{array}$ & $\begin{array}{l}268 \\
(88.3)\end{array}$ & $\begin{array}{l}324 \\
(93.6)\end{array}$ & $\begin{array}{l}362 \\
(96.8)\end{array}$ & 13.7 & $\begin{array}{l}288 \\
(69.1)\end{array}$ & $\begin{array}{l}246 \\
(78.8)\end{array}$ & $\begin{array}{l}288 \\
(83.9)\end{array}$ & $\begin{array}{l}358 \\
(96.8)\end{array}$ & 27.7 \\
\hline & Dire Dawa & $\begin{array}{l}198 \\
(57.6)\end{array}$ & $\begin{array}{l}155 \\
(52.9)\end{array}$ & $\begin{array}{l}242 \\
(57.2)\end{array}$ & $\begin{array}{l}317 \\
(87.4)\end{array}$ & 29.8 & $\begin{array}{l}110 \\
(33.5)\end{array}$ & $\begin{array}{l}98 \\
(27.7)\end{array}$ & $\begin{array}{l}194 \\
(40.3)\end{array}$ & $\begin{array}{l}246 \\
(56.7)\end{array}$ & 23.2 \\
\hline \multirow[t]{2}{*}{ Place of residence } & Urban & $\begin{array}{l}930 \\
(66.6)\end{array}$ & $\begin{array}{l}784 \\
(68.9)\end{array}$ & $\begin{array}{l}1186 \\
(76)\end{array}$ & $\begin{array}{l}1385 \\
(90.1)\end{array}$ & 23.5 & $\begin{array}{l}655 \\
(34)\end{array}$ & $\begin{array}{l}615 \\
(44.4)\end{array}$ & $\begin{array}{l}944 \\
(50.8)\end{array}$ & $\begin{array}{l}1256 \\
(80.1)\end{array}$ & 46.1 \\
\hline & Rural & $\begin{array}{l}1377 \\
(21.6)\end{array}$ & $\begin{array}{l}1316 \\
(23.7)\end{array}$ & $\begin{array}{l}1729 \\
(26.4)\end{array}$ & $\begin{array}{l}2668 \\
(51.3)\end{array}$ & 29.7 & $\begin{array}{l}144 \\
(2.3)\end{array}$ & $\begin{array}{l}192 \\
(2.6)\end{array}$ & $\begin{array}{l}322 \\
(4.0)\end{array}$ & $\begin{array}{l}1443 \\
(21.2)\end{array}$ & 18.9 \\
\hline \multirow[t]{4}{*}{ Mother education } & No education & $\begin{array}{l}1321 \\
(21)\end{array}$ & $\begin{array}{l}1133 \\
(21.7)\end{array}$ & $\begin{array}{l}1387 \\
(25.4)\end{array}$ & $\begin{array}{l}1932 \\
(53.3)\end{array}$ & 32.3 & $\begin{array}{l}217 \\
(2.5)\end{array}$ & $\begin{array}{l}214 \\
(2.3)\end{array}$ & $\begin{array}{l}360 \\
(4.6)\end{array}$ & $\begin{array}{l}965 \\
(17.2)\end{array}$ & 14.7 \\
\hline & Primary & $\begin{array}{l}502 \\
(48)\end{array}$ & $\begin{array}{l}480 \\
(39.4)\end{array}$ & $\begin{array}{l}1090 \\
(45.5)\end{array}$ & $\begin{array}{l}1316 \\
(73)\end{array}$ & 25 & $\begin{array}{l}204 \\
(10.4)\end{array}$ & $\begin{array}{l}193 \\
(8.5)\end{array}$ & $\begin{array}{l}523 \\
(15.4)\end{array}$ & $\begin{array}{l}978 \\
(38.6)\end{array}$ & 28.2 \\
\hline & Secondary & $\begin{array}{l}447 \\
(71.7)\end{array}$ & $\begin{array}{l}428 \\
(80.9)\end{array}$ & $\begin{array}{l}276 \\
(85.5)\end{array}$ & $\begin{array}{l}502 \\
(92.3)\end{array}$ & 20.6 & $\begin{array}{l}341 \\
(45)\end{array}$ & $\begin{array}{l}342 \\
(57.7)\end{array}$ & $\begin{array}{l}235 \\
(72.4)\end{array}$ & $\begin{array}{l}462 \\
(78.4)\end{array}$ & 33.4 \\
\hline & Higher & $\begin{array}{l}37 \\
(97.4)\end{array}$ & $\begin{array}{l}59 \\
(92.2)\end{array}$ & $\begin{array}{l}162 \\
(90.9)\end{array}$ & $\begin{array}{l}303 \\
(98)\end{array}$ & 0.6 & $\begin{array}{l}37 \\
(90.2)\end{array}$ & $\begin{array}{l}58 \\
(90.6)\end{array}$ & $\begin{array}{l}148 \\
(74.1)\end{array}$ & $\begin{array}{l}294 \\
(93.2)\end{array}$ & 3 \\
\hline \multirow[t]{4}{*}{ Father education } & No education & $\begin{array}{l}956 \\
(21.1)\end{array}$ & $\begin{array}{l}747 \\
(19.6)\end{array}$ & $\begin{array}{l}945 \\
(24.6)\end{array}$ & $\begin{array}{l}1349 \\
(43.0)\end{array}$ & 21.9 & $\begin{array}{l}119 \\
(2.6)\end{array}$ & $\begin{array}{l}115 \\
(3.0)\end{array}$ & $\begin{array}{l}200 \\
(5.2)\end{array}$ & $\begin{array}{l}637 \\
(20.3)\end{array}$ & 17.7 \\
\hline & Primary & $\begin{array}{l}554 \\
(37.2)\end{array}$ & $\begin{array}{l}598 \\
(36.3)\end{array}$ & $\begin{array}{l}1189 \\
(42.7)\end{array}$ & $\begin{array}{l}1300 \\
(60.2)\end{array}$ & 23 & $\begin{array}{l}155 \\
(10.3)\end{array}$ & $\begin{array}{l}168 \\
(10.2)\end{array}$ & $\begin{array}{l}484 \\
(17.3)\end{array}$ & $\begin{array}{l}839 \\
(38.8)\end{array}$ & 28.5 \\
\hline & Secondary & $\begin{array}{l}599 \\
(68.1)\end{array}$ & $\begin{array}{l}575 \\
(65.4)\end{array}$ & $\begin{array}{l}412 \\
(69.5)\end{array}$ & $\begin{array}{l}580 \\
(77.9)\end{array}$ & 9.8 & $\begin{array}{l}369 \\
(41.1)\end{array}$ & $\begin{array}{l}381 \\
(43.4)\end{array}$ & $\begin{array}{l}308 \\
(51.9)\end{array}$ & $\begin{array}{l}508 \\
(68.2)\end{array}$ & 27.1 \\
\hline & Higher & $\begin{array}{l}161 \\
(77.4)\end{array}$ & $\begin{array}{l}151 \\
(83.0)\end{array}$ & $\begin{array}{l}331 \\
(72.4)\end{array}$ & $\begin{array}{l}528 \\
(82.3)\end{array}$ & 4.9 & $\begin{array}{l}117 \\
(28.2)\end{array}$ & $\begin{array}{l}122 \\
(76.2)\end{array}$ & $\begin{array}{l}231 \\
(62.3)\end{array}$ & $\begin{array}{l}438 \\
(77.0)\end{array}$ & 48.8 \\
\hline \multirow[t]{5}{*}{ Wealth index } & Poorest & & $\begin{array}{l}202 \\
(12.7)\end{array}$ & $\begin{array}{l}439 \\
(17)\end{array}$ & $\begin{array}{l}888 \\
(47.5)\end{array}$ & 47.5 & & $\begin{array}{l}27 \\
(0.7)\end{array}$ & $85(1.7)$ & $\begin{array}{l}347 \\
(11)\end{array}$ & 11 \\
\hline & Poorer & & $\begin{array}{l}247 \\
(18.6)\end{array}$ & $\begin{array}{l}346 \\
(23.7)\end{array}$ & $\begin{array}{l}615 \\
(55.8)\end{array}$ & 55.8 & & $\begin{array}{l}21 \\
(1.3)\end{array}$ & $55(2.9)$ & $\begin{array}{l}350 \\
(20.8)\end{array}$ & 20.8 \\
\hline & Middle & & $\begin{array}{l}315 \\
(25.2)\end{array}$ & $\begin{array}{l}370 \\
(27)\end{array}$ & $\begin{array}{l}540 \\
(62.6)\end{array}$ & 62.6 & & $\begin{array}{l}28 \\
(1.9)\end{array}$ & $49(3.2)$ & $\begin{array}{l}325 \\
(24.2)\end{array}$ & 24.2 \\
\hline & Richer & & $\begin{array}{l}356 \\
(30.6)\end{array}$ & $\begin{array}{l}468 \\
(35.4)\end{array}$ & $\begin{array}{l}547 \\
(67.1)\end{array}$ & 67.1 & & $\begin{array}{l}66 \\
(4.5)\end{array}$ & $\begin{array}{l}111 \\
(7.4)\end{array}$ & $\begin{array}{l}351 \\
(28.5)\end{array}$ & 28.5 \\
\hline & Richest & & $\begin{array}{l}980 \\
(58)\end{array}$ & $\begin{array}{l}1292 \\
(74.9)\end{array}$ & $\begin{array}{l}1463 \\
(84.8)\end{array}$ & 84.8 & & $\begin{array}{l}665 \\
(26.6)\end{array}$ & $\begin{array}{l}966 \\
(45.6)\end{array}$ & $\begin{array}{l}1326 \\
(70.3)\end{array}$ & 70.3 \\
\hline \multirow[t]{2}{*}{$\begin{array}{l}\text { Mother work } \\
\text { status }\end{array}$} & No working & $\begin{array}{l}1035 \\
(34.0)\end{array}$ & $\begin{array}{l}1474 \\
(29.6)\end{array}$ & $\begin{array}{l}1799 \\
(34)\end{array}$ & $\begin{array}{l}2688 \\
(53.4)\end{array}$ & 19.4 & $\begin{array}{l}408 \\
(13.2)\end{array}$ & $\begin{array}{l}519 \\
(10.4)\end{array}$ & $\begin{array}{l}721 \\
(13.6)\end{array}$ & $\begin{array}{l}1695 \\
(33.7)\end{array}$ & 20.5 \\
\hline & Working & $\begin{array}{l}1269 \\
(30.7)\end{array}$ & $\begin{array}{l}626 \\
(39.3)\end{array}$ & $\begin{array}{l}1114 \\
(45.7)\end{array}$ & $\begin{array}{l}1365 \\
(63.2)\end{array}$ & 32.5 & $\begin{array}{l}389 \\
(9.4)\end{array}$ & $\begin{array}{l}288 \\
(18.1)\end{array}$ & $\begin{array}{l}544 \\
(22.3)\end{array}$ & $\begin{array}{l}1004 \\
(46.5)\end{array}$ & 37.1 \\
\hline \multirow[t]{2}{*}{$\begin{array}{l}\text { Sex of household } \\
\text { head }\end{array}$} & Male & $\begin{array}{l}1841 \\
(30.9)\end{array}$ & $\begin{array}{l}1709 \\
(30.6)\end{array}$ & $\begin{array}{l}2245 \\
(36.3)\end{array}$ & $\begin{array}{l}3128 \\
(55.8)\end{array}$ & 24.9 & $\begin{array}{l}582 \\
(9.7)\end{array}$ & $\begin{array}{l}573 \\
(10.2)\end{array}$ & $\begin{array}{l}900 \\
(14.5)\end{array}$ & $\begin{array}{l}2037 \\
(36.7)\end{array}$ & 27 \\
\hline & Female & $\begin{array}{l}466 \\
(38.0)\end{array}$ & $\begin{array}{l}391 \\
(39.4)\end{array}$ & $\begin{array}{l}670 \\
(42.9)\end{array}$ & $\begin{array}{l}925 \\
(58.2)\end{array}$ & 20.2 & $\begin{array}{l}217 \\
(17.5)\end{array}$ & $\begin{array}{l}234 \\
(23.6)\end{array}$ & $\begin{array}{l}366 \\
(23.4)\end{array}$ & $\begin{array}{l}662 \\
(41.6)\end{array}$ & 24.1 \\
\hline \multirow[t]{3}{*}{ Mother age } & $15-19$ & $\begin{array}{l}155 \\
(28.8)\end{array}$ & $\begin{array}{l}135 \\
(27.3)\end{array}$ & $\begin{array}{l}152 \\
(33.5)\end{array}$ & $\begin{array}{l}207 \\
(66.8\end{array}$ & 38 & $66(6)$ & $\begin{array}{l}55 \\
(6.8)\end{array}$ & $57(96)$ & $\begin{array}{l}161 \\
(32.8)\end{array}$ & 26.8 \\
\hline & $20-34$ & $\begin{array}{l}1647 \\
(28)\end{array}$ & $\begin{array}{l}1530 \\
(29.1)\end{array}$ & $\begin{array}{l}2156 \\
(35.5)\end{array}$ & $\begin{array}{l}2976 \\
(64)\end{array}$ & 36 & $\begin{array}{l}596 \\
(6.1)\end{array}$ & $\begin{array}{l}611 \\
(5.8)\end{array}$ & $\begin{array}{l}1015 \\
(10.9)\end{array}$ & $\begin{array}{l}1989 \\
(27.8)\end{array}$ & 21.7 \\
\hline & $35-49$ & $\begin{array}{l}505 \\
(21.3)\end{array}$ & $\begin{array}{l}435 \\
(22.7)\end{array}$ & $\begin{array}{l}607 \\
(27)\end{array}$ & $\begin{array}{l}870 \\
(52.8)\end{array}$ & 31.5 & $\begin{array}{l}137 \\
(3.7)\end{array}$ & $\begin{array}{l}141 \\
(3.8)\end{array}$ & $\begin{array}{l}194 \\
(5.9)\end{array}$ & $\begin{array}{l}549 \\
(23.1)\end{array}$ & 19.4 \\
\hline \multirow[t]{3}{*}{ Marital status } & $\begin{array}{l}\text { Never married/ } \\
\text { divorced/widowed }\end{array}$ & $\begin{array}{l}280 \\
(37.6)\end{array}$ & $\begin{array}{l}199 \\
(37.5)\end{array}$ & $\begin{array}{l}297 \\
(41.4)\end{array}$ & $\begin{array}{l}313 \\
(58.9)\end{array}$ & 21.3 & $\begin{array}{l}142 \\
(18.9)\end{array}$ & $\begin{array}{l}105 \\
(19.8)\end{array}$ & $\begin{array}{l}166 \\
(23.1)\end{array}$ & $\begin{array}{l}251 \\
(47.3)\end{array}$ & 28.4 \\
\hline & $\begin{array}{l}\text { Married/living } \\
\text { together }\end{array}$ & $\begin{array}{l}2027 \\
(31.5)\end{array}$ & $\begin{array}{l}1901 \\
(31.4)\end{array}$ & $\begin{array}{l}2618 \\
(37.3)\end{array}$ & $\begin{array}{l}3740 \\
(56.1)\end{array}$ & 24.6 & $\begin{array}{l}657 \\
(10.1)\end{array}$ & $\begin{array}{l}702 \\
(11.6)\end{array}$ & $\begin{array}{l}1100 \\
(15.6)\end{array}$ & $\begin{array}{l}2448 \\
(36.7)\end{array}$ & 26.6 \\
\hline & 1 & & & & & 24.7 & & & & & 28.8 \\
\hline
\end{tabular}


Table 2 (continued)

\begin{tabular}{|c|c|c|c|c|c|c|c|c|c|c|c|}
\hline \multirow[t]{2}{*}{$\begin{array}{l}\text { Background } \\
\text { characteristics }\end{array}$} & \multirow[t]{2}{*}{ Categories } & \multicolumn{4}{|c|}{$\begin{array}{l}\text { Number (percent) of women received } \\
\text { prenatal care }\end{array}$} & \multirow[t]{2}{*}{$\begin{array}{l}\text { \% Change during } \\
\text { 2000-2016 }\end{array}$} & \multicolumn{4}{|c|}{$\begin{array}{l}\text { Number (percent) of women received with } \\
\text { skilled birth attendant }\end{array}$} & \multirow[t]{2}{*}{$\begin{array}{l}\text { \% Change during } \\
\text { 2000-2016 }\end{array}$} \\
\hline & & 2000 & 2005 & 2011 & 2016 & & 2000 & 2005 & 2011 & 2016 & \\
\hline \multirow[t]{4}{*}{$\begin{array}{l}\text { Birth in the last of } \\
\text { five years }\end{array}$} & & $\begin{array}{l}1473 \\
(36.5)\end{array}$ & $\begin{array}{l}1357 \\
(36.4)\end{array}$ & $\begin{array}{l}1979 \\
(44.2)\end{array}$ & $\begin{array}{l}2619 \\
(61.2)\end{array}$ & & $\begin{array}{l}614 \\
(15.1)\end{array}$ & $\begin{array}{l}638 \\
(17.1)\end{array}$ & $\begin{array}{l}994 \\
(22.2)\end{array}$ & $\begin{array}{l}1877 \\
(43.9)\end{array}$ & \\
\hline & 2 & $\begin{array}{l}716 \\
(26.2)\end{array}$ & $\begin{array}{l}656 \\
(26.5)\end{array}$ & $\begin{array}{l}799 \\
(29.4)\end{array}$ & $\begin{array}{l}1217 \\
(50.2)\end{array}$ & 24 & $\begin{array}{l}157 \\
(5.7)\end{array}$ & $\begin{array}{l}152 \\
(6.1)\end{array}$ & $\begin{array}{l}234 \\
(8.6)\end{array}$ & $\begin{array}{l}702 \\
(28.9)\end{array}$ & 23.2 \\
\hline & 3 & $\begin{array}{l}108 \\
(28.3)\end{array}$ & $\begin{array}{l}74 \\
(21.6)\end{array}$ & $\begin{array}{l}126 \\
(24.8)\end{array}$ & $\begin{array}{l}200 \\
(44.7)\end{array}$ & 16.4 & $\begin{array}{l}25 \\
(6.5)\end{array}$ & $\begin{array}{l}16 \\
(4.7)\end{array}$ & $37(7.2)$ & $\begin{array}{l}105 \\
(23.5)\end{array}$ & 17 \\
\hline & 4 & $\begin{array}{l}10 \\
(29.4)\end{array}$ & $\begin{array}{l}13 \\
(40.0)\end{array}$ & $\begin{array}{l}11 \\
(23.9)\end{array}$ & $\begin{array}{l}16 \\
(40.0)\end{array}$ & 10.6 & $\begin{array}{l}3 \\
(10.0)\end{array}$ & $4(3.3)$ & $1(0.1)$ & $\begin{array}{l}14 \\
(35.0)\end{array}$ & 25 \\
\hline \multirow[t]{3}{*}{ Parity } & 1 & $\begin{array}{l}578 \\
(42.1)\end{array}$ & $\begin{array}{l}523 \\
(42.5)\end{array}$ & $\begin{array}{l}786 \\
(53.3)\end{array}$ & $\begin{array}{l}1042 \\
(70.9)\end{array}$ & 28.8 & $\begin{array}{l}323 \\
(23.2)\end{array}$ & $\begin{array}{l}329 \\
(26.7)\end{array}$ & $\begin{array}{l}520 \\
(35.2)\end{array}$ & $\begin{array}{l}868 \\
(59.0)\end{array}$ & 35.8 \\
\hline & $2-4$ & $\begin{array}{l}1033 \\
(34.8)\end{array}$ & $\begin{array}{l}984 \\
(34.8)\end{array}$ & $\begin{array}{l}1338 \\
(39.6)\end{array}$ & $\begin{array}{l}1854 \\
(60.0)\end{array}$ & 25.2 & $\begin{array}{l}339 \\
(11.3)\end{array}$ & $\begin{array}{l}372 \\
(13.1)\end{array}$ & $\begin{array}{l}570 \\
(16.8)\end{array}$ & $\begin{array}{l}1230 \\
(39.8)\end{array}$ & 28.5 \\
\hline & $5+$ & $\begin{array}{l}696 \\
(24.6)\end{array}$ & $\begin{array}{l}593 \\
(23.5)\end{array}$ & $\begin{array}{l}791 \\
(27.4)\end{array}$ & $\begin{array}{l}1157 \\
(43.9)\end{array}$ & 19.3 & $\begin{array}{l}137 \\
(4.8)\end{array}$ & $\begin{array}{l}106 \\
(4.2)\end{array}$ & $\begin{array}{l}176 \\
(6.1)\end{array}$ & $\begin{array}{l}601 \\
(22.8)\end{array}$ & 18 \\
\hline \multirow[t]{2}{*}{ Distance } & Big problem & & $\begin{array}{l}1062 \\
(23.5)\end{array}$ & $\begin{array}{l}1655 \\
(29.8)\end{array}$ & $\begin{array}{l}1009 \\
(43.3)\end{array}$ & 43.3 & & $\begin{array}{l}279 \\
(6.2)\end{array}$ & $\begin{array}{l}486 \\
(8.7)\end{array}$ & $\begin{array}{l}596 \\
(26.8)\end{array}$ & 26.8 \\
\hline & Not a big problem & & $\begin{array}{l}1038 \\
(50.4)\end{array}$ & $\begin{array}{l}1257 \\
(54.4)\end{array}$ & $\begin{array}{l}3044 \\
(61.3)\end{array}$ & 61.3 & & $\begin{array}{l}528 \\
(25.7)\end{array}$ & $\begin{array}{l}778 \\
(35.5)\end{array}$ & $\begin{array}{l}2103 \\
(42.3)\end{array}$ & 42.3 \\
\hline
\end{tabular}

Table 3

Multilevel multivariable analysis of factors associated with prenatal care service utilization, in Ethiopia 2000-2016.

\begin{tabular}{|c|c|c|c|c|c|c|c|c|}
\hline \multirow{2}{*}{$\frac{\text { Variables }}{\text { Residence (urban) }}$} & \multicolumn{2}{|l|}{2000} & \multicolumn{2}{|l|}{2005} & \multicolumn{2}{|l|}{2011} & \multicolumn{2}{|l|}{2016} \\
\hline & OR & $95 \% \mathrm{CI}$ & OR & $95 \% \mathrm{CI}$ & OR & $95 \% \mathrm{CI}$ & OR & $95 \% \mathrm{CI}$ \\
\hline \multicolumn{9}{|c|}{ women education (no. educ) } \\
\hline Primary & 2.184 & $1.841-2.590$ & 1.412 & $1.197-1.665$ & 1.679 & $1.470-1.917$ & 1.435 & $1.238-1.664$ \\
\hline Secondary & 4.065 & $2.973-5.558$ & 2.670 & $1.942-3.6703$ & 3.502 & $2.314-5.299$ & 2.249 & $1.615-3.131$ \\
\hline Higher & 6.437 & $2.077-13.07$ & 3.229 & $1.172-8.894$ & 4.128 & $2.094-8.136$ & 4.092 & $2.063-8.118$ \\
\hline \multicolumn{9}{|l|}{ Wealth (poorest) } \\
\hline Poorer & - & - & 1.415 & $1.1406-1.756$ & 1.419 & $1.193-1.687$ & 1.955 & $1.647-2.322$ \\
\hline Middle & - & - & 1.964 & $1.588-2.429$ & 1.695 & $1.423-2.019$ & 2.001 & $1.665-2.405$ \\
\hline Richer & - & - & 2.362 & $1.904-2.928$ & 2.093 & $1.761-2.489$ & 2.655 & $2.183-3.228$ \\
\hline Richest & - & - & 3.429 & $2.678-4.391$ & 4.058 & $3.215-5.124$ & 3.292 & $2.522-4.295$ \\
\hline \multicolumn{9}{|l|}{ Insurance (no) } \\
\hline Yes & - & - & & & 0.928 & $0.374-2.308$ & 1.049 & $0.7472-1.474$ \\
\hline \multicolumn{9}{|l|}{ Partner's education (no. educ) } \\
\hline Primary & 1.478 & $1.276-1.710$ & 1.359 & $1.169-1.581$ & 1.338 & $1.178-1.519$ & 1.313 & $1.145-1.505$ \\
\hline Secondary & 2.027 & $1.643-2.501$ & 1.957 & $1.578-2.427$ & 1.745 & $1.365-2.229$ & 1.512 & $1.192-1.918$ \\
\hline Higher & 3.568 & $1.975-6.446$ & 2.464 & $1.399-4.342$ & 1.847 & $1.315-2.594$ & 1.246 & $0.904-1.717$ \\
\hline \multicolumn{9}{|l|}{ Working status (no) } \\
\hline Yes & 0.979 & $0.867-1.106$ & 1.171 & $1.015-1.350$ & 1.266 & $1.122-1.428$ & 1.086 & $0.950-1.242$ \\
\hline \multicolumn{9}{|l|}{ Women age (15-19) } \\
\hline $20-34$ & 0.913 & $0.7018-1.188$ & 1.1267 & $0.860-1.475$ & 1.264 & $0.976-1.636$ & 1.043 & $0.779-1.395$ \\
\hline $35-49$ & 0.905 & $0.662-1.237$ & 1.057 & $0.764-1.463$ & 1.207 & $0.893-1.633$ & 0.855 & $0.614-1.192$ \\
\hline \multicolumn{9}{|l|}{ Parity (1) } \\
\hline $2-4$ & 1.153 & $0.953-1.395$ & 0.967 & $0.791-1.183$ & 0.955 & $0.798-1.143$ & 0.812 & $0.664-0.993$ \\
\hline $5+$ & 1.057 & $0.844-1.322$ & 0.795 & $0.627-1.008$ & 0.872 & $0.705-1.077$ & 0.706 & $0.561-0.887$ \\
\hline \multicolumn{9}{|l|}{ Distance (a big problem) } \\
\hline Not a big problem & & & 1.700 & $1.486-1.945$ & 1.308 & $1.148-1.490$ & 1.232 & $1.084-1.402$ \\
\hline Access to media (no) & - & - & & & & & & \\
\hline Yes & 1.333 & $1.061-1.674$ & 1.092 & $0.975-2.283$ & 1.692 & $1.181-2.425$ & 1.402 & $1.017-1.932$ \\
\hline \multicolumn{9}{|l|}{ Women autonomy (low) } \\
\hline High & 2.619 & $1.391-4.932$ & 2.109 & $1.269-3.504$ & 1.527 & $1.118-2.088$ & 2.098 & $1.157-3.804$ \\
\hline Middle & 1.416 & $0.583-3.439$ & 1.292 & $0.827-2.018$ & 1.516 & $1.108-2.075$ & 1.090 & $0.729-1.629$ \\
\hline _cons & & & & & & & 2.083 & $1.206-3.598$ \\
\hline \multicolumn{9}{|l|}{$\begin{array}{l}\text { Random-effects parameters } \\
\text { region: Identity }\end{array}$} \\
\hline sd (_cons) & 0.917 & $0.599-1.403$ & 1.040 & $0.678-1.596$ & 1.018 & $0.662-1.567$ & 1.073 & $0.696-1.655$ \\
\hline ICC & 0.043 & & 0.079 & & 0.055 & & 0.104 & \\
\hline AIC & 7236. & & 6454.1 & & 7984. & & 7291. & \\
\hline BIC & 7394. & & 6637.3 & & 8199.8 & & 7468. & \\
\hline Log-likelihood & -359 & & -3200 & & -3961 & & -361 & \\
\hline Deviance & 7188. & & 6400.1 & & 7922. & & 7239. & \\
\hline LRT & 783.7 & alue $=<0.0001)$ & 671.67 & $\mathrm{ue}=<0.0001)$ & 789.58 & $<0.0001)$ & 953.9 & lue $=<0.0001)$ \\
\hline
\end{tabular}

analysis outcomes to assess factors associated with prenatal care utilization. The result revealed residence, maternal education, household wealth index, partner's education, distance were steadily significantly associated with prenatal health care utilization from 2000 to 2016 .
Higher wealth status was significantly associated with prenatal care utilization, with women in the rich or middle household's economic status (highest wealth index) having significantly higher odds of prenatal care utilization relative to those in the poorest (lowest wealth 
index) families (Table 3 ). Throughout all surveys, this inequality between middle or rich and poor wealth index appeared to increase significantly.

There is a consistent adverse association concerning the maternal education level and prenatal care utilization: the higher a woman has educated, the more likely she could utilize prenatal care. In 2000-2016 surveys, women who attended primary, secondary and higher education had higher odds of prenatal care utilization than those with no education (p-value $<0.001$ ).

Furthermore, overall time women whose partners' had a primary, secondary, and higher education level had greater odds of prenatal care utilization than those with no education ( $\mathrm{p}$-value $<0.001$ ).

Regarding residence type, comparatively rural women are consistently less likely to utilize the prenatal care service from 2000 to 2016. Another predictor that was consistently significantly associated with prenatal care utilization was the distance (getting medical help for self). The odds of prenatal care were statistically significantly higher among women who had not got medical assistance for themselves in 2000, 2005, 2011, and 2016 Ethiopian DHS survey.

Working mothers have a greater odds of prenatal care in 2005 and 2011 surveys (OR $=1.17$; 95\%CI: $1.015-1.350$ ) in 2005 and (OR = 1.26; 95\%CI: $1.122-1.428$ ) in 2011, while this inequality was not statistically significant in 2000 and 2016 surveys. On the contrary, mothers with
$2-4$, and at least five children ever born had a lower odds of prenatal care utilization $(\mathrm{OR}=0.81$; 95\%CI: $0.664-0.993)$ and $(\mathrm{OR}=0.71 ; 95 \%$ CI: 0.561-0.887), respectively in 2016, whereas this disparity was not statistically significant from 2000 to 2011 .

\subsection{Multivariable analysis: factors associated with a skilled birth assistant}

The multivariable multilevel logistic regression results factors associated with a skilled birth assistant were presented in Table 4 . The results showed that overall time, women who lived in rural areas had decreases in the odds of a skilled birth assistant compared to women living in urban areas. Further, there is a disparity in skilled birth assistance utilization by sex of household head. Women heads of household had significantly greater odds of a skilled birth assistant in 2000 and 2005 , but this figure is not statistically significant in the recent two surveys (2011 and 2016).

Maternal education was another maternally related characteristic consistently statistically significantly associated with a skilled birth assistant. Primary education, secondary education, and higher education levels had significantly greater odds of a skilled birth assistant overall. Similarly, the highest odds of skilled birth assistants were among women whose partners with at least a primary education level in the last fifteen

Table 4

Multilevel multivariable analysis of factors associated with skilled birth attendant service utilization, in Ethiopia $2000-2016$.

\begin{tabular}{|c|c|c|c|c|c|c|c|c|}
\hline \multirow[t]{2}{*}{ Variables } & \multicolumn{2}{|l|}{2000} & \multicolumn{2}{|l|}{2005} & \multicolumn{2}{|l|}{2011} & \multicolumn{2}{|l|}{2016} \\
\hline & OR & $95 \% \mathrm{CI}$ & OR & $95 \% \mathrm{CI}$ & OR & $95 \% \mathrm{CI}$ & OR & $95 \% \mathrm{CI}$ \\
\hline \multicolumn{9}{|l|}{ Residence (urban) } \\
\hline Rural & 0.1066 & $0.079-0.1405$ & 0.302 & $0.218-0.418$ & 0.208 & $0.159-0.271$ & 0.269 & $0.209-0.346$ \\
\hline \multicolumn{9}{|c|}{ Woman education (no. educ) } \\
\hline Primary & 2.257 & $1.720-2.963$ & 1.675 & $1.264-2.221$ & 1.636 & $1.329-2.014$ & 1.518 & $1.297-1.777$ \\
\hline Secondary & 3.775 & $2.738-5.205$ & 2.614 & $1.844-3.704$ & 3.611 & $2.452-5.318$ & 2.426 & $1.785-3.295$ \\
\hline Higher & 20.504 & $5.784-72.680$ & 6.951 & $2.546-18.978$ & 5.061 & $2.812-9.106$ & 4.100 & $2.306-7.287$ \\
\hline \multicolumn{9}{|l|}{ Hh sex (male) } \\
\hline Female & 1.389 & $1.049-1.839$ & 1.441 & $1.075-1.932$ & 1.212 & $0.959-1.531$ & 1.197 & $0.998-1.437$ \\
\hline \multicolumn{9}{|l|}{ Wealth (poorest) } \\
\hline Poorer & - & - & 1.004 & $0.545-1.847$ & 1.221 & $0.834-1.788$ & 2.214 & $1.809-2.708$ \\
\hline Middle & - & - & 1.562 & $0.879-2.774$ & 1.059 & $0.716-1.566$ & 2.358 & $1.906-2.917$ \\
\hline Richer & - & - & 3.252 & $1.971-5.364$ & 1.658 & $1.188-2.313$ & 3.047 & $2.446-3.795$ \\
\hline Richest & - & - & 5.353 & $3.225-8.886$ & 3.383 & $2.371-4.825$ & 4.446 & $3.405-5.805$ \\
\hline \multicolumn{9}{|l|}{ Insurance (no) } \\
\hline Yes & - & - & - & - & 2.019 & $0.870-4.684$ & 1.996 & $1.418-2.810$ \\
\hline \multicolumn{9}{|c|}{ Partner's education (no. educ) } \\
\hline Primary & 2.122 & $1.575-2.859$ & 1.553 & $1.140-2.115$ & 1.461 & $1.167-1.829$ & 1.206 & $1.032-1.408$ \\
\hline Secondary & 2.984 & $2.177-4.090$ & 2.573 & $1.847-3.586$ & 2.434 & $1.781-3.326$ & 2.129 & $1.672-2.713$ \\
\hline Higher & 5.747 & $3.375-9.785$ & 3.425 & $1.924-6.097$ & 2.355 & $1.598-3.468$ & 1.977 & $1.440-2.716$ \\
\hline \multicolumn{9}{|l|}{ Working status (no) } \\
\hline Yes & 0.963 & $0.771-1.206$ & 1.206 & $0.943-1.542$ & 1.075 & $0.889-1.299$ & 1.099 & $0.948-1.275$ \\
\hline \multicolumn{9}{|l|}{ Woman age (15-19) } \\
\hline $20-34$ & 0.780 & $0.513-1.185$ & 1.642 & $1.063-2.538$ & 1.809 & $1.212-2.701$ & 0.859 & $0.629-1.173$ \\
\hline $35-49$ & 1.059 & $0.616-1.822$ & 2.508 & $1.427-4.407$ & 1.789 & $1.102-2.907$ & 0.867 & $0.603-1.247$ \\
\hline \multicolumn{9}{|l|}{ Parity (1) } \\
\hline $2-4$ & 0.610 & $0.454-0.821$ & 0.385 & $0.283-0.524$ & 0.499 & $0.391-0.638$ & 0.560 & $0.453-0.692$ \\
\hline $5+$ & 0.517 & $0.344-0.775$ & 0.209 & $0.136-0.323$ & 0.398 & $0.286-0.554$ & 0.452 & $0.351-0.583$ \\
\hline \multicolumn{9}{|c|}{ Distance (a big problem) } \\
\hline Not a big problem & - & - & 1.378 & $1.085-1.748$ & 1.722 & $1.422-2.085$ & 1.199 & $1.027-1.400$ \\
\hline \multicolumn{9}{|l|}{ Access to media (no) } \\
\hline Yes & 1.156 & $0.889-1.504$ & 1.289 & $0.912-1.82$ & 1.483 & $1.070-2.054$ & 2.27 & $1.051-4.904$ \\
\hline \multicolumn{9}{|c|}{ Woman autonomy (low) } \\
\hline High & 1.219 & $0.764-2.147$ & 1.61 & $1.063-2.435$ & 1.559 & $1.138-2.138$ & 2.759 & $1.397-4.041$ \\
\hline Middle & 1.206 & $0.764-2.147$ & 1.49 & $1.029-2.158$ & 1.411 & $1.074-1.853$ & 1.793 & $1.004-2.729$ \\
\hline Intercept & & & & & & & .814 & $0.453-1.462$ \\
\hline \multirow{2}{*}{\multicolumn{9}{|c|}{$\begin{array}{l}\text { Random-effects parameters } \\
\text { region: Identity }\end{array}$}} \\
\hline & & & & & & & & \\
\hline sd (_cons) & 1.336 & $0.876-2.038$ & 1.388 & $0.909-2.119$ & 1.277 & $0.837-1.948$ & 1.274 & $0.831-1.954$ \\
\hline ICC & 0.081 & & 0.129 & & 0.112 & & 0.128 & \\
\hline AIC & 2562.83 & & 2420 . & & 3613. & & 5938. & \\
\hline BIC & 2714.15 & & 2624. & & 3829. & & 6115. & \\
\hline Log-likelihood & -1259. & & -1180 & & -177 & & -294 & \\
\hline Deviance & 2518.83 & & 2360 . & & 3551. & & 5886. & \\
\hline LRT & 1313.57 & alue $=<0.0001)$ & 1202. & value $=<0.0001)$ & 1313. & value $=<0.0001)$ & 1345. & value $=<0.0001)$ \\
\hline
\end{tabular}


years (Table 2).

Also, the household wealth index was significantly associated with a skilled birth assistant. Women who belong to the richer or the richest wealth index had an increased odd of a skilled birth assistant, compared to the poorest wealth index in the overall period after controlling for other covariates. Women with not a big problem of getting medical help for themselves had a statistically significantly higher odds of utilizing skilled birth assistant from 2005 to 2016, compared to women with no a big problem of getting medical help for self after controlling for other covariates.

Women who had covered health insurance were less more likely to use skilled birth assistance (OR $=1.99$; 95\%CI: 1.42-2.81) in 2016. Compared to younger women (less than 20 years), those women who are in the range 20-34 and 35-49 had a greater odds of using skilled birth assistance during delivery (OR $=1.64$; 95\% CI: $1.06-2.54$, OR $=2.51$; 95\% CI: 1.43-4.41) in 2005 and (OR $=1.81$; 95\% CI: $1.21-2.70$, OR = 1.79; 95\% CI: 1.10-2.91) in 2011surveys, respectively. Nevertheless, there was no statistically significant difference in maternal age and likelihood of utilizing skilled birth assistance in 2000 and 2016 surveys. There were statistically significant differences in the SBA use of parity. The women who had parity 2-4 and at least five were less likely to use SBA across all surveys.

\subsection{Model goodness of fit}

In this study, two-level structures with mothers as first-level units and regions as second-level units have been used. Mothers selected randomly from the same region are assumed to share more similar characters than mothers chosen randomly from the different regions. Three multilevel logistic regression models: an empty model with random intercept, random intercept with the fixed effects model and random coefficient with a random intercept model has been fitted. In the first step, the heterogeneity of proportions between regions in a multilevel analysis was tested. A test statistic for assessing heterogeneity in the proportion of individuals among regions yields the chi-square test (p-value $<0.0001$ ) for each survey, implying there is evidence of heterogeneity among regions. The hypothesis, there is no cross-regional variation in women's PNC and SBA utilization was observed as significant (p-value $<0.0001$ ). Therefore, there is evidence of cross-regional variation in women's prenatal care services and skilled birth attendants. Further, the Wald test statistic is significant (p-value $<0.05$ ), concludes that there is a significant variation in PNC and SBA use among women.

The intra correlation coefficient (ICC) was used to measure the variation in prenatal care incidence and skilled birth attendant explained by grouping women in the regions. The random intercept model's deviance and covariates added were compared and found that the random intercept with a fixed slope model was better than the random intercept only model. Furthermore, the AIC and BIC values ensure that the smaller AIC and BIC values for the random intercept with fixed slope models. The variance of the random effect of the intercept and fixed slope model decreased compared to the random effects of the empty intercept model. The reduction of the variance of random effect of the intercept model is due to the enclosure of fixed independent variables. Considering the fixed predictors can provide additional predictive value on the incidence of PNC and SBA services in each region.

\section{Discussion}

This study analyzed the trends and associated factors with PNC and SBA utilization using the 2000-2016 Ethiopian DHS. Despite the fact, the use of PNC service increased by $10 \%$, and SBA service increased by almost four times from the year 2000-2016, in Ethiopia, the utilization of these services was still low compared to other countries. For instance, the prevalence of PNC utilization was $92 \%$ in Kenya in $2014,{ }^{23} 68.5 \%$ in Ghana, ${ }^{24}$ but greater than the finding of a study from South Sudan ${ }^{25}$ which reported only $42 \%$ and nearly consistent with a result from Benin $^{26}$ and Nigeria ${ }^{27}$ which reported $61 \%$ and $64 \%$ respectively. Also, the SBA prevalence was lower than a finding of Nigeria, ${ }^{28}$ which reported $65.5 \%$, Kenya, ${ }^{23}$ which reported $65 \%$, and Ghana, ${ }^{24}$ which reported $83.6 \%$. Afar, Oromia, and Somali regions had a minimal PNC and SBA service utilization prevalence than other regions in the last four surveys. These disparities might be due to differences in cultures from peoples in these regions who have similar cultures and traditions, even though equal economic status. The current study suggests vital confirmation that no maternal health care service utilization before and during birth has continued a primary public problem despite minor improvements.

Furthermore, woman and partner's education, higher wealth index, living in urban, female household head, lower parity, woman age greater than 20, working, covered health insurance, and media access were significant correlates for the disparity in a greater utilization of PNC and SBA services in Ethiopia from 2000 to 2016.

Maternal education was found to be the strongest predictor of both PNC and SBA utilization in Ethiopia. Our study found that mothers educated were consistently more likely to utilize prenatal health care compared with those who had no education at all. Similar finding from Northwest Ethiopia, ${ }^{29}$ Gura Dhamole Woreda, Bale zone, southeast Ethiopia, ${ }^{30}$ Nigeria, ${ }^{28}$ Jordan, ${ }^{31}$ Afghanistan, ${ }^{32}$ Angola, ${ }^{33}$ a systematic review of sub-Saharan Africa, ${ }^{34}$ and evidence from on 32 low-income countries across Asia, Sub-Saharan Africa and Latin America Harminder Guliani et al. ${ }^{35}$ reported a higher odds of PNC utilization among educated women relative to uneducated. It might be that educated women can make decisions about their health and find quality health care that helps them get better care for their own and the newborn. Therefore, we ought to encourage women to pursue their education to a higher education level to have a tangible change in seeking maternal health care.

Similarly, we identified partners' education level was a significant factor for utilizing PNC and SBA, increased likelihood using PNC and SBA as the education their partners' had a higher education level. Previous studies from Afghanistan, ${ }^{32}$ and Nigeria $^{28}$ also found that low maternal and partner education levels were associated with a lower likelihood of using maternal health care services. A systematic review and meta-analysis of Tesfalidet et al. ${ }^{19}$ in Ethiopia reported that the significantly increased odds (OR $=1.49,95 \% \mathrm{CI}=1.32-1.69)$ of antenatal care utilization among women whose partners were educated.

In our study, it was found significantly lower odds of PNC and SBA utilization among women from the rural and as compared to the rural residents. Conversely, women who belong to the highest household wealth index demonstrated higher levels of using PNC and SBA than their poorest counterparts. It is congruent with the results of other studies reported higher odds of prenatal care utilization among urban women in the Southwest Shoa Zone, Ethiopia, ${ }^{36}$ Benin, ${ }^{26}$ Nigeria, ${ }^{28}$ and Afghanistan, ${ }^{32}$ Angola, ${ }^{33}$ Uganda, ${ }^{37}$ Lao $^{38}$ and Malawi ${ }^{18}$ more than women from rural areas.

Similar findings from Nigeria, ${ }^{39}$ China, ${ }^{40}$ Uganda, ${ }^{37}$ Lao, ${ }^{38}$ Benin, ${ }^{26}$ Malawi, ${ }^{18}$ and Ghana ${ }^{13}$ also report the increased likelihood of utilizing maternal health care services with increasing household wealth index. The highest odds of using PNC and SBA services were among women in the richer and the richest wealth index category (Table 2). Women who belong to a higher wealth index were almost five (OR $=4.73,95 \% \mathrm{CI}$ : 1.98-11.30) times more likely to utilize SBA than those belonging to the lower wealth index in Malawi. ${ }^{18}$ Furthermore, Calistus et al. ${ }^{36}$ in the Southwest Shoa Zone, Ethiopia and Ababaw et al., ${ }^{21}$ in Northwest Ethiopia reported women in richest wealth were 8.94 (OR $=8.9495 \%$ CI: $2.45-32.61$ ), and 1.21 (OR $=1.2195 \% \mathrm{CI}$ : $(0.71,2.05)$ times more like to utilize SBA care compared to the poorest wealth, respectively. One possible explanation might be the lack of access to health care services in rural areas because of the distance (unreachability of health facilities and professionals), lack of transportation services, and poor infrastructure. It might be because urban women were more likely to get 
health facilities near their home and better access media about health education and the benefits of maternal health care utilization. Nevertheless, in Ethiopia, since the vast majority of the people, about $85 \%$, lived in rural areas, PNC and SBA's level remains very low. Further, the poorest women might not be able to cover costs for the services.

Our study also found that a big problem of getting medical help for self has persisted impact on PNC and SBA utilization in Ethiopia, despite a minor change in the odds of using the services. For instance, only $43 \%$ and $26.8 \%$ of women had a considerable distance to a health facility of getting medical help for self-use PNC and SBA services in 2016. Edite et al., ${ }^{33}$ using Dande Health and Demographic Surveillance System in Angola, found that women living at a greater distance of a health facility were less likely to use maternal health care. Further, Gizachew et al. ${ }^{30}$ found that traveling for less than $1 \mathrm{~h}$ and $1-2 \mathrm{~h}$ to get health facilities was associated with four times and two times more like to use SBA services compared more than $2 \mathrm{~h}$ to get health facilities for medical help. One challenging factor might be a big distance from health facilities. The poorest wealth index tends to have the lowest prevalence of seeking prenatal care and skilled birth attendant during pregnancy and delivery time since most of the women delivered outside of health facilities.

Moreover, parity of 2-4 and more than five children ever born was found significantly associated with lower odds utilizing PNC in the recent 2016 DHS and utilizing SBA from 2000 to 2016 in Ethiopia. It was in agreement with Ruo Zhang et al., ${ }^{40}$ and a systematic review of sub-Saharan countries. ${ }^{34}$ A lower prevalence of the PNC and SBA observed among higher parity might be that despite the economic status, the larger family size, the more household expenditure, and more subjected to unwanted last pregnancy.

Women's access to media also saw the disparity in PNC and SBA use. The study demonstrates that the significant increasing progress of the PNC and SBA has made it within the women who had access to media. It is similar to a finding from Benin, ${ }^{26}$ India, ${ }^{15}$ where women who had access to media are more likely to utilize maternal health care services. Similarly, Markos et al., ${ }^{14}$ using DHS 2000-2011 in Ethiopia, found that access to media was significantly associated with higher health care utilization odds. In this situation, our findings suggest the importance of further access to media, ensuring access to reproductive and health care education about mothers and newborns.

The study also found that women who had covered health insurance almost two times more likely to use skilled birth attendants. It is similar to a finding from Ghana ${ }^{13}$ and India. ${ }^{20}$

We also found that a woman's autonomy (a woman's freedom to make decisions independently, participate in critical decisions and exercise more control in her household and life) was a significant predictor of PNC and SBA. A similar analysis of the Afghanistan $\mathrm{DHS}^{32}$ and Nigeria DHS $^{28,41}$ found that high autonomy was associated with greater odds of utilizing maternal health care services. On the other hand, Sanni et al., ${ }^{26}$ based on a population-based cross-sectional study of Benin DHS, found that women's decision-making power is an essential predictor of prenatal care service utilization. Taking the low autonomy as a reference, high (OR $=1.22$; 95\%CI, 1.01-1.47) and autonomy levels were significantly associated with greater odds of using prenatal care services, but they found no significant association between skilled birth assistance and women's autonomy.

In this study, women aged 20-34 and 35-49 and currently working were found significant predictors of higher prevalence of prenatal care utilization. Similar research from the Southwest Shoa Zone, Ethiopia, ${ }^{36}$ China, ${ }^{40}$ Nigeria, ${ }^{28}$ and Benin ${ }^{26}$ reported a higher age of women at birth and currently working, significantly affecting maternal health care services utilization. In contrast, Ranjana et al., $2019^{15}$ in India reported currently working was inversely associated with utilizing maternal health care services. Further, our analysis found that a woman household head was significantly associated with higher odds of using skilled birth attendants. Similar to our findings, Sanni et al. ${ }^{26}$ in Benin found that the female household head was associated with higher odds of skilled delivery assistant care utilization.

\section{Limitations}

Some of the limitations noted in this study are first; the data are secondary from mothers' recall of past events, subject to recall bias since the recall period is long (five years preceding the survey). The irregularities between the questionnaires are also another prime limit of this study. Further, some important variables are missing due to too much missing value.

\section{Conclusion}

Overall, even though Ethiopia was making dramatic progress over the last fifteen years in increasing the prevalence of maternal health care services utilization, the figures are still lower. Particularly the utilization of skilled birth attendant service is very low. It should be improved to achieve the sustainable development goals to minimize the maternal mortality rates and early neonatal deaths by 2030 . Moreover, we found that a higher level of women's and partner's education, higher household wealth index, female household head, older age, and covered health insurance were predictor factors of greater odds of utilizing PNC and SBA services. Being a rural resident and higher parity were also significant predictors of lower odds of PNC and SBA utilization. Therefore, improving women and partners' education and household wealth is vital for the health policies aiming to increase PNC and SBA services utilizations in Ethiopia.

Further, the disparities in utilizing PNC and SBA services across regions vary even through equal socioeconomic groups. Therefore, to sustain the progress equals more attention is needed for Afar, Somali, and Oromia regions to increase maternal health care services utilization in Ethiopia.

\section{Ethics approval and consent to participate}

Not applicable.

\section{Consent for publication}

Not applicable.

\section{Availability of data and material}

The data that support the findings of this study are available from the corresponding author upon reasonable request.

\section{Funding}

This research received no financial support for the research, authorship, and/or publication of this article.

\section{Authors' contributions}

MAA conceived the original idea of the study, design the study, analyzed the data and drafted the manuscript. BTW conceived the original idea of the study, involved with the conception of the study, statistical analysis, interpretation and revision of the manuscript. All authors read and approved the final manuscript.

\section{Declaration of competing interest}

The authors declare that they have no competing interests.

\section{Acknowledgments}

The authors are most grateful to the DHS Measure Program for providing the data for the study. 


\section{Abbreviations}

AIC Akaike Information Correction

BIC Bayesian Information Correction

CSA Central Statistical Agency

CI Confidence Interval

DHS Demographic and Health Survey

EDHS Ethiopian Demographic and Health Survey

HSTP Health Sector Transformation Plan

ICC Intra Correlation Coefficient

ICF Inner City Fund

LRT Likelihood Ratio Test

$\mathrm{MOH} \quad$ Ministry of Health

OR Odds Ratio

PNC Prenatal Care

SBA Skilled Birth Attendant

SDGs Sustainable Development Goals

SNNPR South Nations Nationalities and People Region

UFMR Under Five Mortality Rate

UN United Nation

USAID United State Agency for International Development

WHO World Health Organization

\section{Appendix A. Supplementary data}

Supplementary data to this article can be found online at https://doi. org/10.1016/j.cegh.2021.100771.

\section{References}

1 World Health Organization, Unicef, Unfpa, World Bank Group and the United Nations Population Division Geneva. Trends in Maternal Mortality: 2000 to 2017. World Health Organization; 2019.

2 Mekonnen Tensae, Dune Tinashe, Perz Janette. Maternal health service utilization of adolescent women in sub-Saharan Africa: a systematic scoping review. BMC Pregnancy Childbirth. 2019;19:366.

3 World Health Organization Geneva. Maternal Mortality. World Health Organization; 2019, 2019.

4 Central Statistical Agency [Ethiopia] and ICF. Ethiopia Demographic and Health Survey 2016; CSA [Ethiopia] and ICF: Addis Ababa. 2016. Ethiopia; Rockville, MD, USA.

5 Federal Democratic Republic of Ethiopia Ministry of Health. Health Sector Transformation Plan 2015/16-2019/20. 2015. Addis Ababa, Ethiopia.

6 Tesfaye Gezahegn, Loxton Deborah, Chojenta Catherine, Assefa Nega, Smith Roger. Magnitude, trends and causes of maternal mortality among reproductive aged women in Kersa health and demographic surveillance system, eastern Ethiopia. BMC Wom Health. 2018;18:198.

7 Central Statistical Agency [Ethiopia] and ORC Macro. Ethiopia Demographic and Health Survey 2000; Central Statistical Authority and ORC Macro: Addis Ababa, Ethiopia., MD, USA: Calverton; 2000.

8 Central Statistical Agency [Ethiopia] and ICF. Ethiopia Demographic and Health Survey 2005; CSA [Ethiopia] and ICF: Addis Ababa, Ethiopia. MD, USA: Calverton; 2005.

9 Central Statistical Agency [Ethiopia] and Inner City Fund International. Ethiopia Demographic and Health Survey 2011; CSA [Ethiopia] and ICF: Addis Ababa, Ethiopia., MD, USA: Calverton; 2011.

10 Word Health Organization. Standards for Improving Quality of Maternal and Newborn Care in Health Facilities Geneva. World Health Organization; 2016.

11 United Nations. Transforming Our World: The 2030. New York: Agenda for Sustainable Development; 2015.

12 Mekonnen Tensae, Dune Tinashe, Perz Janette, Felix Akpojene Ogbo. Trends and determinants of antenatal care service use in Ethiopia between 2000 and 2016. Int J Environ Res Publ Health. 2019;16:748.

13 Jacob Novignon, Ofori Bernice, Gyabaa Tabiri Kwasi, Pulok Mohammad H. Socioeconomic inequalities in maternal health care utilization in Ghana. Int J Equity Health. 2019;18:141.

14 Mezmur Markos, Kannan Navaneetham, Letamo Gobopamang, Bariagaber Hadgu. Socioeconomic inequalities in the uptake of maternal healthcare services in Ethiopia. BMC Health Serv Res. 2017;17:367.

15 Singh Ranjana, Neogi Sutapa B, Hazra Avishek, et al. Utilization of maternal health services and its determinants: a cross-sectional study among women in rural Uttar Pradesh, India. J Health Popul Nutr. 2019;38:13.

16 Alam Nazmul, Hajizadeh Mohammad, Dumont Alexandre, Fournier Pierre. Inequalities in maternal health care utilization in sub-saharan african countries: a multiyear and multi-country analysis. PloS One. 2015;10(4), e0120922.
17 Ali Balhasan, Chauhan Shekhar. Inequalities in the utilisation of maternal health care in rural India: evidences from national family health survey III \& IV. BMC Publ Health. 2020;20:369.

18 Kazanga Isabel, Munthali Alister C, McVeigh Joanne, Mannan Hasheem, MacLachlan Malcolm. Predictors of utilisation of skilled maternal healthcare in lilongwe district, Malawi. Int J Health Pol Manag. 2019;8(12):700-710.

19 Tekelab Tesfalidet, Chojenta Catherine, Smith Roger, Loxton Deborah. Factors affecting utilization of antenatal care in Ethiopia: a systematic review and metaanalysis. PloS One. 2019;14(4), e0214848.

20 Kumar Gunjan, Choudhary Tarun S, Srivastava Akanksha, et al. Utilisation, equity and determinants of full antenatal care in India: analysis from the National Family Health Survey 4. BMC Pregnancy Childbirth. 2019;19:327.

21 Worku Abebaw G, Yalew Alemayehu W, Afework Mesganaw F. Factors affecting utilization of skilled maternal care in Northwest Ethiopia: a multilevel analysis. BMC Int Health Hum Right. 2013;13:20.

22 Ononokpono Dorothy N, Odimegwu Clifford O. Determinants of maternal health care utilization in Nigeria: a multilevel approach. Pan African Medical Journal. 2014;17 (Supp 1):2.

23 Banke-Thomas A, Banke-Thomas O, Kivuvani M, Ameh C. Maternal health services utilisation by Kenyan adolescent mothers: analysis of the demographic health survey 2014. Sex and Reprod Healthcare. 2017;12:37-46.

24 Nuamah Gladys B, Agyei-Baffour Peter, Mensah Kofi A, et al. Access and utilization of maternal healthcare in a rural district in the forest belt of Ghana. BMC Pregnancy Childbirth. 2019;19:6.

25 Ngatho S Mugo, Dibley Michael J, E Agho Kingsley. Prevalence and risk factors for non-use of antenatal care visits: analysis of the 2010 South Sudan household survey. BMC Pregnancy Childbirth. 2015;15:68.

26 Yaya Sanni, Uthman Olalekan A, Amouzou Agbessi, Ekholuenetale Michael, Bishwajit Ghose. Inequalities in maternal health care utilization in Benin: a population based cross-sectional study. BMC Pregnancy Childbirth. 2018;18:194.

27 Nwosu Chijioke O, Ataguba John E. Socioeconomic inequalities in maternal health service utilisation: a case of antenatal care in Nigeria using a decomposition approach. BMC Publ Health. 2019;19:1493.

28 Ogbo Felix A, Trinh Felicity F, Ahmed Kedir Y, et al. Prevalence, trends, and drivers of the utilization of unskilled birth attendants during democratic governance in Nigeria from 1999 to 2018. Int J Environ Res Publ Health. 2020;17:372.

29 Alemayehu Mulunesh, Mekonnen Wubegzier. The prevalence of skilled birth attendant utilization and its correlates in north west Ethiopia. BioMed Res Int. 2015; 2015:1-8. Article ID 436938.

30 Gizachew S Ayele, Abulie T Melku, Semere S Belda. Utilization of skilled birth attendant at birth and associated factors among women who gave birth in the last 24 months preceding the survey in Gura Dhamole Woreda, Balezone, southeast Ethiopia. BMC Publ Health. 2019;19:1501.

31 Heba H Hijazi1, Mohammad S Alyahya1, Amer M Sindiani, Saqan Rola S, Abdulhakeem M Okour. Determinants of antenatal care attendance among women residing in highly disadvantaged communities in northern Jordan: a cross-sectional study. Reprod Health. 2018;15:106.

32 Mumtaz Sarwat, Bahk Jinwook, Khang Young-Ho. Current status and determinants of maternal healthcare utilization in Afghanistan: analysis from Afghanistan Demographic and Health Survey 2015. PloS One. 2019;14(6), e0217827.

33 Rosario Edite VN, Gomes Manuel C, Brito Miguel, Costa Diogo. Determinants of maternal health care and birth outcome in the Dande Health and Demographic Surveillance System area, Angola. PloS One. 2019;14(8), e0221280.

34 Ijeoma N Okedo-Alex, Ifeyinwa C Akamike, Obumneme B Ezeanosike, Chigozie J Uneke. Determinants of antenatal care utilisation in sub-Saharan Africa: a systematic review. BMJ Open. 2019;9, e031890.

35 Guliani Harminder, Sepehri Ardeshir, Serieux John. Determinants of prenatal care use: evidence from 32 low-income countries across Asia, Sub-Saharan Africa and Latin America. Health Pol Plann. 2014;29:589-602.

36 Wilunda Calistus, Quaglio Gianluca, Putoto Giovanni, et al. Ana Pilar Betrán, Andrea Atzori. Determinants of utilisation of antenatal care and skilled birth attendant at delivery in South West Shoa Zone, Ethiopia: a cross sectional study. Reprod Health 2015;12:74.

37 Rutaremwa Gideon, Wandera Stephen O, Jhamba Tapiwa, Akiror Edith, Kiconco Angela. Determinants of maternal health services utilization in Uganda. BMC Health Serv Res. 2015;15:271.

38 Do Ngan, Tran Huong TG, Phonvisay Alay, Oh Juhwan. Trends of socioeconomic inequality in using maternal health care services in Lao People's Democratic Republic from year 2000 to 2012. BMC Publ Health. 2018;18:875.

39 Dahiru T, Oche OM. Determinants of antenatal care, institutional delivery and postnatal care services utilization in Nigeria. Pan Africa Medical Journal. 2015;21: 321.

40 Zhang Ruo, Li Shanshan, Li1 Chao, et al. Socioeconomic inequalities and determinants of maternal health services in Shaanxi Province, Western China. PloS One. 2018;13(9), e0202129.

41 Adeniyi F Fagbamigbe, Elizabeth O Hurricane-Ike, Oyindamola B Yusuf, SIdemudia Erhabor. Trends and drivers of skilled birth attendant use in Nigeria (1990-2013): policy implications for child and maternal health. Int J Wom Health. 2017;9:843-853. 